\title{
Effect of prolonged exercise in a hypoxic environment on cardiac function and cardiac troponin $T$
}

\author{
R E Shave, E Dawson, G Whyte, K George, D Gaze, P Collinson
}

Br J Sports Med 2004;38:86-88. doi: 10.1136/bjsm.2002.002832

Background: Exercise induced cardiac fatigue has recently been observed after prolonged exercise. A moderate to high altitude has been suggested as a possible stimulus in the genesis of such cardiac fatigue.

Objective: To investigate if exercise induced cardiac fatigue and or cardiac damage occurs after prolonged exercise in a hypoxic environment.

Methods: Eight trained male triathletes volunteered for the study. Each completed two 50 mile cycle trials, randomly assigned from normobaric normoxia and normobaric hypoxia $\left(15 \% \mathrm{FIO}_{2}\right)$. Echocardiographic assessment and whole blood collection was completed before, immediately after, and 24 hours after exercise. Left ventricular systolic and diastolic functional variables were calculated, and serum was analysed for cardiac troponin T. Results were analysed using a two way repeated measures analysis of variance, with $\alpha$ set at 0.05 .

Results: No significant differences were observed in either systolic or diastolic function across time or between trials. Cardiac troponin T was detected in one subject immediately after exercise in the normobaric hypoxic trial.

Conclusions: A 50 mile cycle trial in either normobaric normoxia or normobaric hypoxia does not induce exercise induced cardiac fatigue. Some people, however, may exhibit minimal cardiac damage after exercise in normobaric hypoxia. The clinical significance of this is yet to be elucidated.

$\mathrm{E}$ xercise induced cardiac fatigue (EICF) has recently been described by many authors, ${ }^{1-4}$ as has minimal cardiac damage after prolonged exercise. ${ }^{5-12}$ Further, it has been suggested that acute altitude exposure may exacerbate the incidence of EICF because of the increased physiological strain associated with exercising at altitude. ${ }^{13}$ Stimulated by the increased participation in endurance events at moderate to high altitude and the adoption by many athletes of normobaric hypoxic training, this study investigated the impact of prolonged exercise in a hypoxic environment on cardiac function and humoral markers of cardiac damage.

\section{METHODS}

Eight trained male volunteers completed the study (mean (SD) age 33.5 (8.8) years, height 1.79 (0.08) m, body mass $\left.77.7(8.3) \mathrm{kg}, \dot{\mathrm{V}}_{2} \mathrm{MAX}, 67.4(6.3) \mathrm{ml} / \mathrm{kg} / \mathrm{min}\right)$. After ethical approval from the universities' ethics committees and before the start of the study, each subject provided written informed consent. The subjects completed two 50 mile cycle trials on a Kingcycle training rig (Kingcycle, High Wycombe, Buckinghamshire, UK), randomly assigned from normobaric normoxia and normobaric hypoxia and separated by 14 days. The trials were completed at an intensity equivalent to lactate threshold (previously determined in normobaric normoxia). Temperature was controlled during both trials $\left(21^{\circ} \mathrm{C}\right)$. The hypoxic trial was completed in a commercially available hypoxic chamber (Edge4 Ltd, London, UK). Within the chamber, the hypoxic environment is generated by a nitrogen dilution technique, which maintains a constant $\mathrm{FIO}_{2}$ of $15 \%$ (simulating an altitude of about $2500 \mathrm{~m}$ ). Subjects were not blinded to the conditions. Echocardiographic assessments and whole blood (venous) collection was completed before the start of exercise, immediately after exercise, and then again 24 hours after exercise, all in normobaric normoxic conditions.

Echocardiographic assessment was completed using a Hewlett-Packard HP Sonos 1000 (2.5 MHz transducer) with simultaneous electrocardiograph recordings. M-Mode images were taken to measure wall and cavity dimensions during both systole and diastole. Variables of systolic function (fractional shortening (FS), stroke volume (SV) and cardiac output ( $\dot{Q})$ ) were calculated using the measurements obtained during M-mode examination. At the time of echocardiographic assessment, blood pressure was measured by standard ausculation techniques. Left ventricular meridional wall stress was calculated as a measure of left ventricular afterload using the formula of Reichek et al. ${ }^{14}$ Pulsed wave Doppler interrogation of mitral valve inflow velocities was performed to assess diastolic function. Peak early filling ( $\mathrm{E}$ wave, $\mathrm{cm}^{-1}$ ) and peak late filling (A wave, $\mathrm{cm}^{-1}$ ) velocities were measured, and the ratio of early to late diastolic filling (E:A) was calculated.

Whole blood samples $(5 \mathrm{ml})$ were drawn from an antecubital vein and allowed to clot. They were then centrifuged, and the serum was drawn off and frozen $\left(-20^{\circ} \mathrm{C}\right)$ for later analysis. Serum samples were assayed for cardiac troponin $\mathrm{T}$ (cTnT) using electrochemiluminesence technology in an Elecsys 1010 automated batch analyser (Roche Diagnostics, Mannheim, Germany).

SV, FS, Q E, A, and E:A were statistically analysed using a two way repeated measures analysis of variance, with $\alpha$ set at 0.05. Differences in completion time were analysed using Student's $t$ tests. cTnT was analysed descriptively.

\section{RESULTS}

Completion times for the normobaric hypoxic and normobaric normoxic trials were not significantly different (mean (SD) 125 (6) $v 126 \quad$ (7) min respectively). No significant differences were observed across time or between trials for SV, FS, E, A, or E:A (table 1). Q was significantly raised immediately after exercise in both trials $(p<0.05)$; no difference was observed between trials. cTnT was increased

Abbreviations: $\mathrm{cTnT}$, cardiac troponin $\mathrm{T} ; \mathrm{EICF}$, exercise induced cardiac fatigue; $F S$, fractional shortening; $\dot{Q}$, cardiac output; SV, stroke volume 
Table 1 Echocardiographic and humoral variables before, after, and 24 hours after exercise in normobaric normoxia and normobaric hypoxia

\begin{tabular}{|c|c|c|c|c|c|c|}
\hline & \multicolumn{3}{|l|}{ Normoxic } & \multicolumn{3}{|l|}{ Hypoxic } \\
\hline & Before & After & $24 \mathrm{~h}$ after & Before & After & $24 \mathrm{~h}$ after \\
\hline $\begin{array}{l}\text { SV (ml) } \\
\text { Q (litres/min) } \\
\text { FS (\%) } \\
\text { E wave }\left(\mathrm{cm}^{-1}\right) \\
\text { A wave }\left(\mathrm{cm}^{-1}\right) \\
\text { E:A } \\
\text { HR (beats/min) } \\
\text { BP (mm Hg) } \\
\quad \text { Systolic } \\
\text { Diastolic } \\
\left.\text { LVMWS (g/ } \mathrm{cm}^{2}\right) \\
\text { LVIDD }(\mathrm{cm}) \\
\text { Myoglobin }(\mu \mathrm{g} / \mathrm{ml}) \\
\text { CK-MB ( } \mathrm{gg} / \mathrm{l}) \\
\text { cTnT (no of positive } \\
\text { results) }\end{array}$ & $\begin{array}{l}115.5(23.3) \\
7.0(2.1) \\
39.3(2.9) \\
80.2(8.7) \\
34.4(8.3) \\
2.4(0.6) \\
60(13) \\
\\
122.2(4.3) \\
79.1(8.3) \\
62.4(7.4) \\
5.3(0.3) \\
39.2(13.2) \\
3.6(2.1) \\
0\end{array}$ & $\begin{array}{l}107.4(25.4) \\
7.7(1.4)^{*} \\
37.5(6.3) \\
77.6(5.6) \\
39.9(3.3) \\
2.0(0.2) \\
73(12) \\
\\
123.4(5.9) \\
79.2(10.4) \\
69.9(18) \\
5.2(0.3) \\
51.5(16.9) \\
3.7(2.0) \\
0\end{array}$ & $\begin{array}{l}107.4(23.7) \\
6.9(2.3) \\
37.4(3.6) \\
81.2(10.2) \\
37.3(9.2) \\
2.3(0.9) \\
64(10) \\
120.9(6.2) \\
78.9(6.0) \\
64.9(4.5) \\
5.3(0.3) \\
52.4(17.5) \\
3.0(1.2) \\
0\end{array}$ & $\begin{array}{l}118.3(24.5) \\
7.6(1.7) \\
37.4(4.4) \\
78.2(8.1) \\
39.6(11.0) \\
2.1(0.5) \\
64(11) \\
123.3(5.9) \\
80.6(7.5) \\
62.7(12.2) \\
5.4(0.4) \\
36.1(7.8) \\
3.2(1.9) \\
0\end{array}$ & $\begin{array}{l}104.6(19.6) \\
8.0(1.3)^{*} \\
33.6(3.9) \\
72.8(11.5) \\
43.9(12.9) \\
1.8(0.4) \\
78(15) \\
122.5(7.1) \\
84.8(5.6) \\
70.1(16.2) \\
5.2(0.4) \\
81.5(38.2) \\
3.3(1.6) \\
1(0.016 \mu \mathrm{g} / \mathrm{l})\end{array}$ & $\begin{array}{l}113.8(19.2) \\
7.3(1.6) \\
37.0(3.3) \\
82.8(12.1) \\
38.6(9.8) \\
2.2(0.6) \\
64(4) \\
120.3(6.5) \\
79.7(5.1) \\
64.6(8.9) \\
5.3(0.3) \\
41.9(13.4) \\
4.6(3.0) \\
0\end{array}$ \\
\hline
\end{tabular}

Values are mean (SD).

*Significantly different from values obtained before exercise $(p<0.05)$.

$H R$, Heart rate; BP, blood pressure; CK-MB, creatine kinase-myocardial band; cTnT, cardiac troponin T; LVIDD, left ventricular internal diameter in diastole; LVMWS, left ventricular meridional wall stress.; FS, fractional shortening; Q, cardiac output; SV, stroke volume; E wave, peak early filling; $A$ wave, peak late filling; $E: A$, early to late diastolic filling.

in one subject $(0.016 \mu \mathrm{g} / \mathrm{l})$ immediately after the normobaric hypoxic trial.

\section{DISCUSSION}

The results of this study suggest that 50 miles of cycling at an intensity equivalent to lactate threshold in either normobaric normoxia or normobaric hypoxia does not induce reductions in either left ventricular systolic or diastolic function. The impact of altered heart rates on serial measurements of diastolic function has been debated. ${ }^{15}{ }^{16}$ In the present study, given that the differences in heart rate were minimal and that echocardiographic measurements were obtained in a supine position (optimising venous return), any effect of altered heart rate on diastolic function would have been minimal. The data from previous studies examining exercise of similar duration in normoxic conditions ${ }^{117} 18$ corroborate the results from the normoxic trial in this study. The additional stimulus of a hypoxic environment did not induce EICF. Although altitude exposure has been previously implicated in the genesis of EICF, ${ }^{13}$ our data suggest that the additional physiological stress of a hypoxic environment during about two hours of exercise is not enough to induce EICF. Whether a hypoxic environment would exacerbate EICF in periods of exercise greater than two hours cannot be ascertained from this study. Future work examining the impact of hypoxia on EICF should use longer exercise protocols. Further, the assessment of left ventricular function during exercise may help to elucidate any alteration in cardiac function during exercise.

Previous studies have investigated cardiomyocyte damage as a possible cause of EICF; therefore we analysed serum for

\section{Take home message}

Two hours of vigorous exercise in either a normobaric hypoxic or normobaric normoxic environment in trained subjects does not produce exercise induced cardiac fatigue. Minimal cTnT release may, however, be observed in some subjects, the long term implications of which are yet to be elucidated.
cTnT. Concomitant with unaltered cardiac function was an absence of cTnT in all samples except one $(0.016 \mu \mathrm{g} / \mathrm{l})$. A cTnT concentration above the detection limit of the assay $(>0.01 \mu \mathrm{g} / \mathrm{l})$ is deemed evidence of cardiac damage; if below $0.1 \mu \mathrm{g} / \mathrm{l}$, it is not suggestive of acute myocardial infarction, but rather represents a level of minor cardiac damage. ${ }^{19}$ Minimal release of cTnT after prolonged exercise has been shown in a limited number of subjects in previous studies. $^{5}{ }^{1120-22}$ The rapid return to baseline cTnT in the one positive subject in our study coupled with the minimal concentration attained may suggest a transient cytosolic leakage propagated by membrane damage, as opposed to cardiomyocyte necrosis. ${ }^{23}{ }^{24}$ It is possible that such cytosolic leakage may be caused by free radical mediated injury, ${ }^{11}$ and as such may explain why the cTnT release in this study was only observed in the normobaric hypoxic trial where free radical production would be increased. ${ }^{25}$ Currently, however, any suggestions of the potential mechanisms responsible for such cTnT release are only speculative. It is noteworthy that the subject who had a raised cTnT concentration was the youngest and least well trained subject. This agrees with previous work suggesting that exercised induced troponin release may be more likely in less well trained athletes. ${ }^{9}$ Hence, it is possible that more subjects may have shown exercised induced troponin release in this study if a less well trained subject pool had been used. At present, however, the precise mechanisms for and clinical significance of minimal cTnT release after prolonged exercise cannot be elucidated.

\section{CONCLUSIONS}

A 50 mile cycle trial at lactate threshold in either normobaric normoxia or normobaric hypoxia does not induce cardiac dysfunction or evidence of cardiac damage in most subjects. Some, however, may show evidence of minimal cardiac damage. Further work is warranted into the factors that may interact to induce minimal cardiac damage in certain people.

\section{Authors' affiliations}

R E Shave, Brunel University, Uxbridge, Middlesex, UK

E Dawson, Manchester Metropolitan University, Manchester, UK

G Whyte, British Olympic Medical Centre, Northwick Park Hospital,

Harrow, Middlesex, UK 
K George, Liverpool John Moores University, Liverpool, UK D Gaze, P Collinson, St Georges Hospital Medical School, London, UK

Correspondence to: Dr Shave, Department of Sport Science, Brunel University, Uxbridge UB8 3PH, Middlesex, UK; rob.shave@brunel.ac.uk

Accepted 14 January 2003

\section{REFERENCES}

1 Perrault $\mathrm{H}$, Peronnet F, Lebeau R, et al. Echocardiographic assessment of left ventricular performance before and after marathon running. Am Heart $J$ 1986;112:1026-31.

2 Douglas PS, O'Toole ML, Hiller WD, et al. Cardiac fatigue after prolonged exercise. Circulation 1987:76:1206-13.

3 Whyte GP, George K, Sharma S, et al. Cardiac fatigue following prolonged endurance exercise of differing distances. Med Sci Sports Exerc 2000;32: 1067-72

4 Haykowsky M, Welsh R, Humen D, et al. Impaired left ventricular systolic function after a half-ironman race. Can J Cardiol 2001;17:687-90.

5 Bonetti A, Tirelli F, Albertini R. Serum cardiac troponin T after repeated endurance exercise events. Int J Sports Med 1996;17:259-62.

6 Davila-Roman VG, Guest TM, Tuteur PG, et al. Transient right but not left ventricular dysfunction after strenuous exercise at high altitude. J Am Coll Cardiol 1997;30:468-73.

7 Denvir MA, Galloway PJ, Meighan AS, et al. Changes in skeletal and cardiac muscle enzymes during the Scottish Coast to Coast Triathlon. Scott Med J 1999:44:49-51.

8 Rifai N, Douglas PS, O'Toole M et al. Cardiac troponin T and I, echocardiographic [correction of electrocardiographic] wall motion analyses, and ejection fractions in athletes participating in the Hawaii Ironman Triathlon. Am J Cardiol 1999;83:1085-9.

9 Neumayr G, Gaenzer H, Pfister R, et al. Plasma levels of cardiac troponin I after prolonged strenuous endurance exercise. Am J Cardiol 2001;87:36971, A10.

10 Siegel AJ, Lewandrowski EL, Chun KY, et al. Changes in cardiac markers including B-natriuretic peptide in runners after the Boston marathon. Am J Cardiol 2001;88:920-3.
11 Neumayr G, Pfister R, Mitterbauer G, et al. Effect of the "Race Across The Alps" in elite cyclists on plasma cardiac troponins I and T. Am J Cardiol 2002;89:484-6.

12 Shave R, Dawson E, Whyte G, et al. Markers of cardiac damage after 2 days of prolonged endurance exercise. J Sports Sci 2002;20:58.

13 Rowe WJ. Endurance exercise and injury to the heart. Sports Med 1993; 16:73-9.

14 Reichek N, Wilson J, St John S, et al. "Noninvasive determination of left ventricular end-systolic stress: validation of the method and initial application." Circulation 1982;65:99-108.

15 Danielsen R, Nordrehaug JE, Vik-Mo H. Importance of adjusting left ventricular diastolic peak filling rate for heart rate. Am J Cardiol 1988:61:489-91.

16 Zoghbi WA, Bolli R. The increasing complexity of assessing diastolic function from ventricular filling dynamics. J Am Coll Cardiol 1991;17:237-8.

17 Lucia A, Serratosa L, Saborido A, et al. Short-term effects of marathon running: no evidence of cardiac dysfunction. Med Sci Sports Exerc 1999:31:1414-21.

18 Goodman JM, McLaughlin PR, Liu PP. Left ventricular performance during prolonged exercise: absence of systolic dysfunction. Clin Sci (Lond) 2001;100:529-37.

19 Collinson PO, Wiggins N, Gaze DC. Clinical evaluation of the ACS:180 cardiac troponin I assay. Ann Clin Biochem 2001;38:509-19.

20 Mair J, Wohlfarter T, Koller A, et al. Serum cardiac troponin T after extraordinary endurance exercise. Lancet 1992;340:1048.

21 Koller A, Mair J, Schobersberger W, et al. Effects of prolonged strenuous endurance exercise on plasma myosin heavy chain fragments and other muscular proteins. Cycling vs running. J Sports Med Phys Fitness 1998;38:10-17.

22 Koller A, Summer P, Moser $\mathrm{H}$. Regular exercise and subclinical myocardial injury during prolonged aerobic exercise. JAMA 1999;282:1816.

23 Mair J, Dienstl F, Puschendorf B. Cardiac troponin T in the diagnosis of myocardial injury. Crit Rev Clin Lab Sci 1992;29:31-57.

24 Chen YJ, Serfass RC, Apple FS. Loss of myocardial CK-MB into the circulation following 3.5 hours of swimming in a rat model. Int J Sports Med 2000;21:561-5.

25 Wozniak A, Drewa G, Chesy G, et al. Effect of altitude training on the peroxidation and antioxidant enzymes in sportsmen. Med Sci Sports Exerc 2001;33:1109-13.

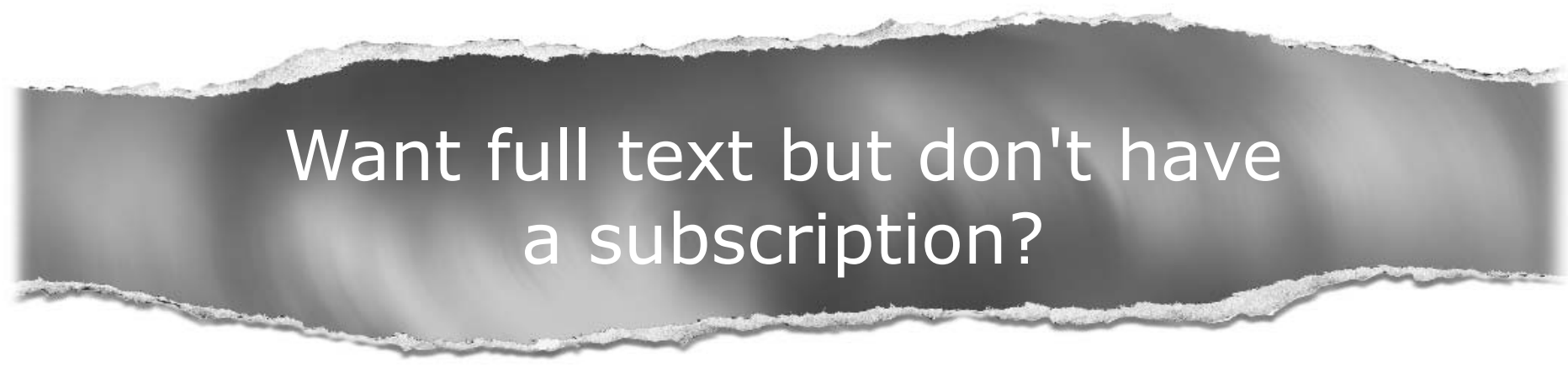

\section{Pay per view}

For just $\$ 8$ you can purchase the full text of individual articles using our secure online ordering service. You will have access to the full text of the relevant article for 48 hours during which time you may

download and print the pdf file for personal use.

\section{www.bjsportmed.com}

